\title{
Probing a light sterile neutrino through heavy charged Higgs boson decays at the LHC
}

\author{
Yi-Lei Tang* \\ School of Physics, KIAS, 85 Hoegiro, Seoul 02455, Republic of Korea
}

(Received 23 April 2018; published 29 August 2018)

\begin{abstract}
We present a $13 \mathrm{TeV}$ proton-proton collider simulation in a $\nu$-two-Higgs-doublet model. The heavy charged Higgs bosons are produced in pairs through the electroweak processes and decay to the light sterile neutrinos (lighter than the $W / Z$ boson masses). The light sterile neutrino further decays into a jet-like object with a muon in it. This helps us discriminate the signal from the backgrounds composed of the standard model jets.
\end{abstract}

DOI: 10.1103/PhysRevD.98.035043

\section{INTRODUCTION}

The seesaw mechanisms [1-5] introduce some righthanded or sterile neutrinos with extremely heavy Majorana masses $\left(\sim 10^{9}-10^{12} \mathrm{GeV}\right)$ to create the light neutrino masses $(\lesssim 0.1 \mathrm{eV}$ according to the oscillation data). However, this sort of model is far beyond the reach of a practical collider. If we reduce the masses down to about $1 \mathrm{TeV}$, the Yukawa coupling among the sterile neutrinos, left-handed neutrinos, and the Higgs doublet are predicted to be so small that it is nearly impossible to produce a practical signal at a collider. In the literature, there are some alternative models within which we can obtain a TeV-scale sterile neutrino as well as a relatively stronger connection with the standard model (SM) sectors. One group of such models utilizes the pseudo-Dirac sterile neutrinos (e.g., Refs. [6-8]) with a relatively larger Yukawa coupling $y$, yet collider searches still involve hunting within an extremely small fraction of $Z^{(*)} / W^{(*)}$-decay products. (See Refs. [9-11] for some experimental results, and Refs. [12-27] for some theoretical approaches.) Another approach at colliders is to associate the seesaw mechanisms with other new physics models [28-35]. We can therefore investigate sterile neutrinos with the aid of other new physics particles.

In this paper, we consider a situation where the charged Higgs boson mainly decays to a sterile neutrino plus a charged lepton. This scenario can be found in some $\nu$-twoHiggs-doublet models ( $\nu$-THDMs) [36-39]. Collider phenomenology has also been studied in the literature as

\footnotetext{
*tangyilei@kias.re.kr
}

Published by the American Physical Society under the terms of the Creative Commons Attribution 4.0 International license. Further distribution of this work must maintain attribution to the author(s) and the published article's title, journal citation, and DOI. Funded by SCOAP . well [40,41]. However, as known, there is a gap in the interesting parameter space where $m_{N} \ll m_{H^{ \pm}}$and $m_{N} \lesssim m_{Z / W}$. In this area, each of the largely boosted sterile neutrinos decays into a single jet-like collimated object, and thus the usual method of analyzing separated objects loses its effectiveness. (For some works on neutrino jets, see Refs. [42,43].) Sometimes, if $m_{N}<m_{W / Z}$, the threebody suppression on the sterile neutrino's decay width will reveal a secondary vertex for us to discriminate the signal (such as that addressed in Ref. [40]). However, the decay length is very sensitive to the model parameters, and it can possibly be well below the 1-.cm scale. Therefore, for a conservative discussion, we neglect all of the secondary vertex information and treat the signal/SM backgrounds in the usual way. Unlike most SM jets, the sterile neutrino jets usually contain leptons. This feature has been applied in, e.g., Refs. $[16,17]$ to search for the lepton jets decayed from the sterile neutrinos. However, we mainly consider hadronic decay and other new physics sectors that participate in the processes in this paper. We also apply this feature to efficiently eliminate the background.

\section{MODEL DESCRIPTION}

Here, we rely on a standard $\nu$-THDM model. The THDM with $\Phi_{i} \rightarrow(-1)^{i-1} \Phi_{i} \quad Z_{2}$ symmetry including the soft-breaking terms is characterized by the effective potential [44]

$$
\begin{aligned}
V= & m_{1}^{2} \Phi_{1}^{\dagger} \Phi_{1}+m_{2}^{2} \Phi_{2}^{\dagger} \Phi_{2}-m_{12}^{2}\left(\Phi_{1}^{\dagger} \Phi_{2}+\Phi_{2}^{\dagger} \Phi_{1}\right) \\
& +\frac{\lambda_{1}}{2}\left(\Phi_{1}^{\dagger} \Phi_{1}\right)^{2}+\frac{\lambda_{2}}{2}\left(\Phi_{2}^{\dagger} \Phi_{2}\right)^{2}+\lambda_{3}\left(\Phi_{1}^{\dagger} \Phi_{1}\right)\left(\Phi_{2}^{\dagger} \Phi_{2}\right) \\
& +\lambda_{4}\left(\Phi_{1}^{\dagger} \Phi_{2}\right)\left(\Phi_{2}^{\dagger} \Phi_{1}\right)+\frac{\lambda_{5}}{2}\left[\left(\Phi_{1}^{\dagger} \Phi_{2}\right)^{2}+\left(\Phi_{2}^{\dagger} \Phi_{1}\right)^{2}\right],
\end{aligned}
$$

where $\Phi_{1,2}$ are the two-Higgs doublets with hypercharge $Y=\frac{1}{2}, \lambda_{1-5}$ are the coupling constants, and $m_{1,2,12}^{2}$ are the 
mass parameters. The $\nu$-THDM is based on the type-I THDM in which all the SM particles $Q_{L}, u_{R}, d_{R}, L_{L}$, and $e_{R}$ couple with the $\Phi_{2}$ field,

$$
\begin{aligned}
\mathcal{L}_{\text {Yukawa }}^{\mathrm{SM}}= & -Y_{u i j} \bar{Q}_{L i} \tilde{\Phi}_{2} u_{R j}-Y_{d i j} \bar{Q}_{L i} \Phi_{2} d_{R j} \\
& -Y_{l i j} \bar{L}_{L i} \Phi_{2} l_{R j}+\text { H.c. }
\end{aligned}
$$

The sterile neutrino together with the left-handed lepton doublets couple with the $\Phi_{1}$ field. In this paper, without loss of generality, we consider only one sterile neutrino $N$. Therefore, the corresponding Lagrangian is given by

$$
\mathcal{L}_{\text {Yukawa }}^{\nu}=-m_{N} \bar{N} N-\left(Y_{i} \bar{L}_{L i} \tilde{\Phi}_{1} N+\text { H.c. }\right),
$$

where $m_{N}$ is the mass of the sterile neutrino and $Y_{i}, i=1$, 2,3 are the Yukawa coupling constants corresponding to the $e, \mu$, and $\tau$ lepton doublets.

In the following discussions, we do not need to care about the details of the electroweak symmetry breaking, nor do we need to discuss the neutral Higgs bosons. After the electroweak symmetry breaking, we acquire the coupling

$$
\mathcal{L} \supset-Y_{i} \sin \beta H^{+} \bar{l}_{i} P_{R} N+\text { H.c. }
$$

where $\tan \beta=\frac{v_{2}}{v_{1}}$ is the ratio of the $\Phi_{1,2}$ vacuum expectation values, and $H^{ \pm}$is the charged Higgs with the mass $m_{H^{ \pm}}$. In the large $\tan \beta \gg 1$ case, Eq. (4) becomes the most significant coupling for $H^{ \pm}$decay, and therefore $H^{ \pm} \rightarrow$ $l^{ \pm} N$ will become the dominant decay channel when $m_{N}<m_{H^{ \pm}}$.

At a proton-proton collider, the $H^{+} H^{-}$pairs can be produced via either the $s$-channel $\gamma^{*}$ and $Z^{*}$, or the off-shell Higgs particles $h^{*}$ and $H^{*}$. The off-shell Higgs particle channel is usually negligible due to the rather small light quark-Higgs coupling constants, except in some special cases when $\lambda_{3}$ or $\lambda_{4}$ is very large. Therefore, in this paper we only consider the $q \bar{q} \rightarrow Z^{*} / \gamma^{*} \rightarrow H^{+} H^{-}$processes. Another important thing that we should mention is the decay length of the sterile neutrino $N$. In fact, the smallness of the left-handed neutrino masses constrains $Y_{i} \cos \beta<10^{-6}$, which in turn amplifies the decay length to $\gtrsim 1 \mathrm{~m}$. This will destroy most of our results discussed in this paper. However, the model we use in this paper is only a simplified model. In reality, this can be quite different in the case of the existence of the other sterile neutrino singlets. For example, in Ref. [45] the appearance of the pseudo-Dirac sterile neutrinos allowed a much larger $Y_{i} \cos \beta \sim 10^{-3}$ in a naturally smaller $\tan \beta \sim 10^{2}-10^{3}$ case, compared with the simplest $\nu$-THDM in which $\tan \beta \gtrsim 10^{4}$. Most of the results of our paper will remain undisturbed in such cases. Furthermore, the current bounds from various experiments on the mixing parameters $\left|U_{i N}\right|^{2} \approx\left(\frac{Y_{i} \cos \beta v}{m_{N}}\right)^{2}$ are no less than $10^{-6}$. In this paper, we only care about the parameter space near these bounds, just as in many theoretical and experimental works in the literature.
Therefore, we do not consider the possibility of the secondary vertex cases.

\section{SIMULATION DETAILS AND RESULTS}

In this paper, we concentrate on the decay $p p \rightarrow Z^{*} /$ $\gamma^{*} \rightarrow H^{+} H^{-}$, with the $H^{ \pm} \rightarrow \mu^{ \pm} N, N \rightarrow \mu^{ \pm} W^{\mp *} \rightarrow \mu^{ \pm} q \bar{q}$ decay chains as shown in Fig. 1. We have chosen the hadronic decay channel of the sterile neutrino because it has the largest branching ratio and it is more convenient for reconstructing the $H^{ \pm}$masses. The muon appearing in the decay products (clustered inside a jet together with the other elements) can help us tag the jets decayed from the sterile neutrinos.

The muons appearing in the decay products can also be replaced by electrons or taus. Here we have chosen the muon channels for the collider's distinctive ability to identify a muon, especially a muon inside of a jet. As for the electron cases, we can estimate the corresponding SM background from the result in this paper due to the lepton universality in the SM. However, one should be aware of the greater difficulties in identifying an electron inside a jet than a muon when attempting to apply our result to the other lepton cases.

The missing energy is predicted to be small in our expected signal. The reducible backgrounds with neutrinos should be eliminated by applying the transverse missing energy (MET) cuts. However, the pileup effects at the future high-luminosity colliders might seriously smear the MET distributions in both the signal and background events, making it difficult to separate them. There are three main sources of reducible backgrounds with neutrinos: from the $\tau^{+} \tau^{-}$'s leptonic decay, from the gauge bosons $W^{+} W^{-} \rightarrow l^{+} \nu l^{-} \bar{\nu}$ decay and the $Z Z \rightarrow \nu \bar{\nu} \mu^{+} \mu^{-}$decay associated with the $b \bar{b}$, and the $t \bar{t} \rightarrow b \bar{b} W^{+} W^{-}$decay. The $\tau^{+} \tau^{-}$s leptonic decay is suppressed by the relatively small branching ratio, and these less energetic muons have a much smaller chance of passing the kinematic cuts. Gauge boson decay channels are further reduced by the higher order of the coupling constants. The only significant reducible channel is $p p \rightarrow t \bar{t} \rightarrow b \bar{b} \mu^{+} \mu^{-} \nu \bar{\nu}$. Therefore, in this paper the only reducible background that we consider is the $t \bar{t}$ channel. DeLPHES with its CMS card shows that the MET distribution of the signal sample is also rather significant even in the case without pileup. This sort of

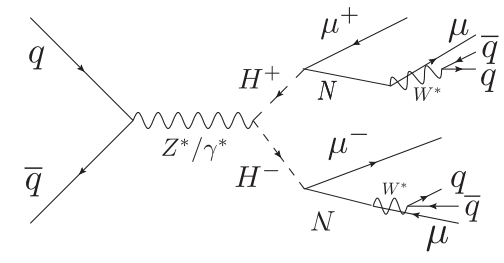

FIG. 1. $\quad p p \rightarrow Z^{*} / \gamma^{*} H^{+} H^{-}$, with the $H^{ \pm} \rightarrow \mu^{ \pm} N, \quad N \rightarrow$ $\mu^{ \pm} W^{\mp *} \rightarrow \mu^{ \pm} q \bar{q}$ decay chains. Since $m_{N} \ll m_{H^{ \pm}}$, the decay products for $N$ will be largely boosted into two jet-like objects. 
MET distribution might be due to the mismeasurement of jet momentum, and usually the direction of the missing energy is parallel to one of the jets; therefore, $\Delta \phi_{\mathrm{MET}-j}$ can be an ideal parameter to cut the reducible background. However, in this paper the jets are from the generator-level particles for a preliminary study, and the future techniques and performances of the missing energy measurements and the influence of pileup effects are unknown. Therefore, in this paper we do not apply the MET cuts during the event selection processes and show our results in two situations: $p p \rightarrow t \bar{t}$ is not considered (called "no- $t \bar{t}$ "), or it is considered (called "with- $t \bar{t}$ "). These indicate the two extreme situations where the reducible backgrounds can or cannot be fully cut out in the future.

We generate our events using the FeYnRules + MADGRAPH5_AMC+PythIA8+DELPHES [46-49] tool chain. The information about the isolated muons come from the DeLPHES results. However, we cluster the generator-level particles (with the isolated muons deducted) by ourselves using FASTJET. The event selection criteria are as follows.

(1) Two isolated muons with at least two $p_{T} \geq 50 \mathrm{GeV}$ and $|\eta|<=5.0$ jets appear. The isolated muons are selected according to the CMS card settings in DELPHES, and the jets are clustered using the anti$k_{T}$ method with the parameter $R=0.9$ using all of the $|\eta|<=5.0$ particles. The two isolated muons do not participate in the jet clustering processes.

(2) The invariant mass between the two muons should be outside the $Z$-boson mass window, $[75,105] \mathrm{GeV}$. The invariant mass between the two $p_{T}$ leading jets should be outside the mass window [60, 130] GeV.

(3) We group the two muons and two jets into two pairs. Each pair contains one muon and one jet. The absolute value of the invariant mass difference should be the smallest among all grouping possibilities (two possibilities for each event). The invariant mass of each pair should be within the range $\left[m_{H^{ \pm}}-50 \mathrm{GeV}, m_{H^{ \pm}}+50 \mathrm{GeV}\right]$.

These are the pure kinematic criteria. The event samples that have only passed these selections have the label "-KIN." For example, "SIG-KIN" labels the signal events that have only passed these selections, while "BKG-KIN" indicates the corresponding background events.
We then tag the jets as the decay product of a sterile neutrino by examining each constituent within the jet. If there is a muon carrying more than $30 \%$ of the total jet energy, then this jet is tagged as a "N-jet." The events containing at least one tagged $\mathrm{N}$-jet are labeled by "- $1 \mathrm{~N}$ jet," and the ones with two tagged $\mathrm{N}$-jets are labeled by "- $2 \mathrm{~N}$-jet." We should also note that we have assumed that $100 \%$ of the muons inside a jet can be perfectly identified by the detector, with no misidentification rate for the other particles. (For some discussions regarding muon identification at the current CMS detector, see Refs. [50-52]. One can refer to the identification and misidentification rates there for a more precise simulation. The authors of Ref. [52] also mentioned identifying a muon inside a $b$ jet.)

Leptons rarely appear in a SM jet, although there are some semileptonic decay channels from a short-lived hadron, especially for $B$ mesons. Both the $b$ jets and the other jets have a non-negligible chance to fake a sterileneutrino jet; however, the probability is so small that it is rather difficult to generate a background "- $2 \mathrm{~N}$-jet" sample. Nevertheless, we can divide the tagged jet number by the total jet number to estimate the "mistagging rate" $R_{\mu}^{j, b}$ for each of the $j j l l$ or bbll samples, with " $j$ " or " $b$ " indicating the $u d c s g$ jets or the $b$ jets, respectively. Finally, we can calculate the $R_{\mu}^{j 2}, R_{\mu}^{b 2}$, or $R_{\mu}^{j} R_{\mu}^{b}$ times "BKG-KIN" cross sections to estimate the two-sterile-neutrino-jet background ("BKG-2N-jet" cross sections).

We classify the background channels according to the final states as well as the mediator resonances and generate the corresponding samples separately. Besides the $p p \rightarrow t \bar{t}$ contributions, the main background channels are $p p \rightarrow \mu^{+} \mu^{-} j j, p p \rightarrow \mu^{+} \mu^{-} j b$, and $p p \rightarrow \mu^{+} \mu^{-} b b$ with no $W / Z$ resonances. The $W / Z$ resonance channels such as $p p \rightarrow Z Z \rightarrow \mu^{+} \mu^{-} j j$ are also considered and analyzed separately. The background cross sections corresponding to different sterile-neutrino-jet number criteria ("-1N-jet" or "-2N-jet") at a $13 \mathrm{TeV}$ proton-proton collider are listed in Tables I and II. In Table I, the $p p \rightarrow t \bar{t} \rightarrow \mu^{+} \mu^{-} b \bar{b} \nu \bar{\nu}$ channels are not included, while in Table II these contributions are included.

Besides the electroweak $p p \rightarrow H^{+} H^{-}$cross sections $\sigma_{p p \rightarrow H^{+} H^{-}}$, the signal cross sections also depend on the

TABLE I. Background cross sections corresponding to each mass window around $m_{H^{ \pm}}$and different sterile-neutrino-jet number criteria. $p p \rightarrow t \bar{t} \rightarrow \mu^{+} \mu^{-} b \bar{b} \nu \bar{\nu}$ contributions are not included.

\begin{tabular}{lccccccc}
\hline \hline$m_{H^{ \pm}} / \mathrm{GeV}$ & 100 & 125 & 150 & 175 & 200 & 225 & 250 \\
\hline$\sigma_{\text {BKG-KIN }} / \mathrm{fb}$ & $1.43 \mathrm{e}+03$ & $1.11 \mathrm{e}+03$ & 730 & 470 & 301 & 193 & 129 \\
$\sigma_{\text {BKG-1N-jet }} / \mathrm{fb}$ & 5.31 & 3.8 & 2.58 & 1.43 & 0.686 & 0.588 & 0.407 \\
$\sigma_{\text {BKG-2N-jet }} / \mathrm{fb}$ & 0.0106 & 0.00858 & 0.00562 & 0.0036 & 0.00239 & 0.00155 & 0.00103 \\
$m_{H^{ \pm}} / \mathrm{GeV}$ & 275 & 300 & 325 & 350 & 375 & 400 & \\
$\sigma_{\text {BKG-KIN }} / \mathrm{fb}$ & 86.3 & 58.2 & 39.3 & 27.9 & 19.1 & 13.3 & \\
$\sigma_{\text {BKG-1N-jet }} / \mathrm{fb}$ & 0.252 & 0.169 & 0.106 & 0.0845 & 0.0609 & 0.0467 & \\
$\sigma_{\text {BKG-2N-jet }} / \mathrm{fb}$ & 0.000617 & 0.000413 & 0.000293 & 0.000213 & 0.000161 & 0.00011 & \\
\hline \hline
\end{tabular}


TABLE II. The same as in Table I, but $p p \rightarrow t \bar{t} \rightarrow \mu^{+} \mu^{-} b \bar{b} \nu \bar{\nu}$ contributions are included.

\begin{tabular}{lccccccc}
\hline \hline$m_{H^{ \pm}} / \mathrm{GeV}$ & 100 & 125 & 150 & 175 & 200 & 225 & 250 \\
\hline$\sigma_{\text {BKG-KIN }} / \mathrm{fb}$ & $2.06 \mathrm{e}+03$ & $1.76 \mathrm{e}+03$ & $1.2 \mathrm{e}+03$ & 724 & 427 & 271 & 181 \\
$\sigma_{\text {BKG-1N-jet }} / \mathrm{fb}$ & 31.4 & 27.8 & 17.3 & 7.74 & 3.56 & 2.51 & 1.57 \\
$\sigma_{\text {BKG-2N-jet }} / \mathrm{fb}$ & 0.183 & 0.186 & 0.134 & 0.0733 & 0.0371 & 0.0228 & 0.0152 \\
$m_{H^{ \pm}} / \mathrm{GeV}$ & 275 & 300 & 325 & 350 & 375 & 400 & \\
$\sigma_{\text {BKG-KIN }} / \mathrm{fb}$ & 121 & 81.2 & 54.3 & 37.5 & 25.6 & 17.9 & \\
$\sigma_{\text {BKG-1N-jet }} / \mathrm{fb}$ & 1 & 0.612 & 0.481 & 0.306 & 0.214 & 0.132 & \\
$\sigma_{\text {BKG-2N-jet }} / \mathrm{fb}$ & 0.0101 & 0.00673 & 0.0044 & 0.00283 & 0.00195 & 0.00138 & \\
\hline \hline
\end{tabular}

TABLE III. The total cross section of $p p \rightarrow H^{+} H^{-}$at a $13 \mathrm{TeV}$ proton-proton collider and the cut efficiencies of the signal corresponding to each mass window on $m_{H^{ \pm}}$and different sterile-neutrino-jet number criteria. The sterile neutrino mass $m_{N}$ is fixed at $10 \mathrm{GeV}$.

\begin{tabular}{lccccccc}
\hline \hline$m_{H^{ \pm}} / \mathrm{GeV}$ & 100 & 125 & 150 & 175 & 200 & 225 & 250 \\
\hline$\sigma_{p p \rightarrow H^{+} H^{-}} / \mathrm{fb}$ & 204 & 91.2 & 47 & 26.5 & 15.9 & 10.1 & 6.65 \\
$R_{\text {SIG-KIN }}$ & 0.154 & 0.159 & 0.16 & 0.212 & 0.214 & 0.208 & 0.206 \\
$R_{\text {SIG-1N-jet }}$ & 0.102 & 0.106 & 0.106 & 0.142 & 0.144 & 0.139 & 0.136 \\
$R_{\text {SIG-2N-jet }}$ & 0.0275 & 0.0284 & 0.0284 & 0.0389 & 0.0379 & 0.0373 & 0.0364 \\
$m_{H^{ \pm}} / \mathrm{GeV}$ & 275 & 300 & 325 & 350 & 375 & 400 & \\
$\sigma_{p p \rightarrow H^{+} H^{-}} / \mathrm{fb}$ & 4.53 & 3.17 & 2.26 & 1.65 & 1.22 & 0.916 & \\
$R_{\text {SIG-KIN }}$ & 0.253 & 0.254 & 0.251 & 0.252 & 0.247 & 0.296 & \\
$R_{\text {SIG-1N-jet }}$ & 0.174 & 0.174 & 0.17 & 0.166 & 0.163 & 0.204 & \\
$R_{\text {SIG-2N-jet }}$ & 0.0498 & 0.0477 & 0.0474 & 0.0461 & 0.0431 & 0.0576 & \\
\hline \hline
\end{tabular}

branching ratios of the $H^{ \pm} \rightarrow \mu^{ \pm} N$ and $N \rightarrow \mu^{ \pm} q \bar{q}$ decay channels. All of these factors can be characterized by one single parameter $\epsilon$, defined by $\sigma_{\mu \text {-channels }}=\epsilon \sigma_{p p \rightarrow H^{+} H^{-}}$, or $\epsilon=\left(\mathrm{Br}_{H^{ \pm} \rightarrow \mu^{ \pm} N} \cdot \mathrm{Br}_{N \rightarrow \mu^{ \pm} q \bar{q}}\right)^{2}$. We calculate the total $p p \rightarrow$ $H^{+} H^{-}$cross sections as well as the cut efficiencies $R_{\text {SIG-X }}$ for each $m_{H^{ \pm}} \in[100,400] \mathrm{GeV}$ in a $25 \mathrm{GeV}$ interval and $m_{N} \in\left[10, \frac{m_{H^{ \pm}}}{10 \mathrm{GeV}}+5\right] \mathrm{GeV}$ in a $2.5 \mathrm{GeV}$ interval. We do not discuss the case of $m_{N}<10 \mathrm{GeV}$ because PythIA8 might not be able to give us reliable showering and hadronization results in this case. Part of the direct result
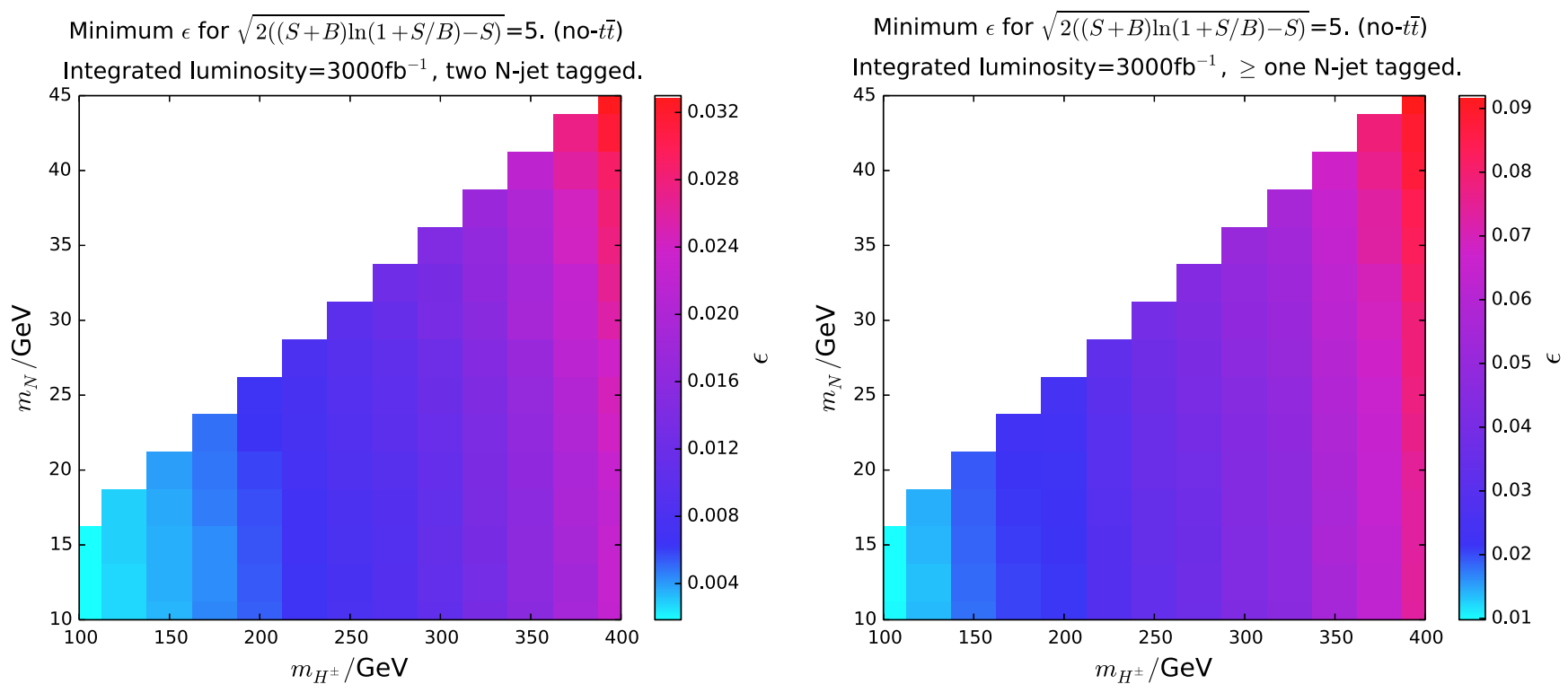

FIG. 2. Minimum $\epsilon$ for $\sqrt{2((S+B) \ln (1+S / B)-S)}=5$. The integrated luminosity is $3 \mathrm{ab}^{-1}$ at a $13 \mathrm{TeV}$ proton-proton collider. $p p \rightarrow t \bar{t} \rightarrow \mu^{+} \mu^{-} b \bar{b} \nu \bar{\nu}$ contributions are not included. 
Minimum $\epsilon$ for $\sqrt{2((S+B) \ln (1+S / B)-S)}=5$. (with-t $\bar{t}$ ) Integrated luminosity $=3000 \mathrm{fb}^{-1}$, two N-jet tagged.

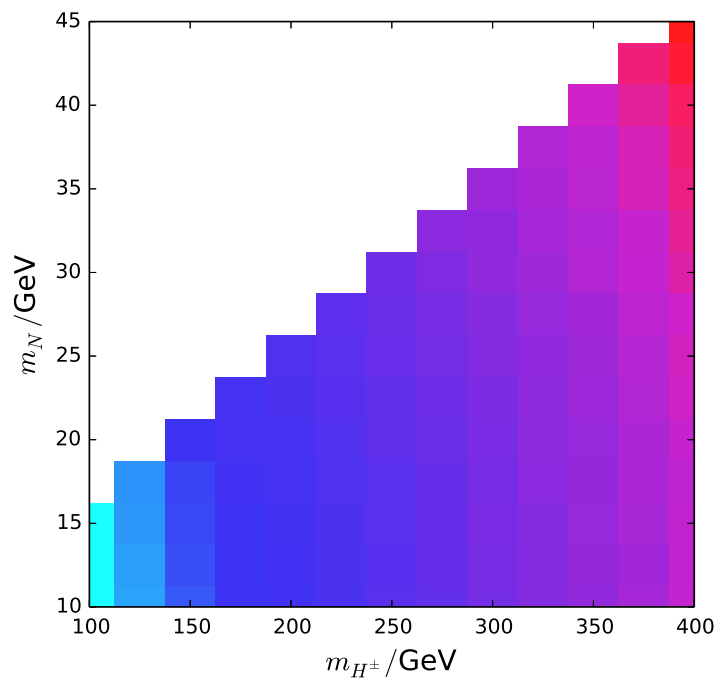

Minimum $\epsilon$ for $\sqrt{2((S+B) \ln (1+S / B)-S)}=5$. (with-t $\bar{t}$ )

Integrated luminosity $=3000 \mathrm{fb}^{-1}, \geq$ one $\mathrm{N}$-jet tagged.

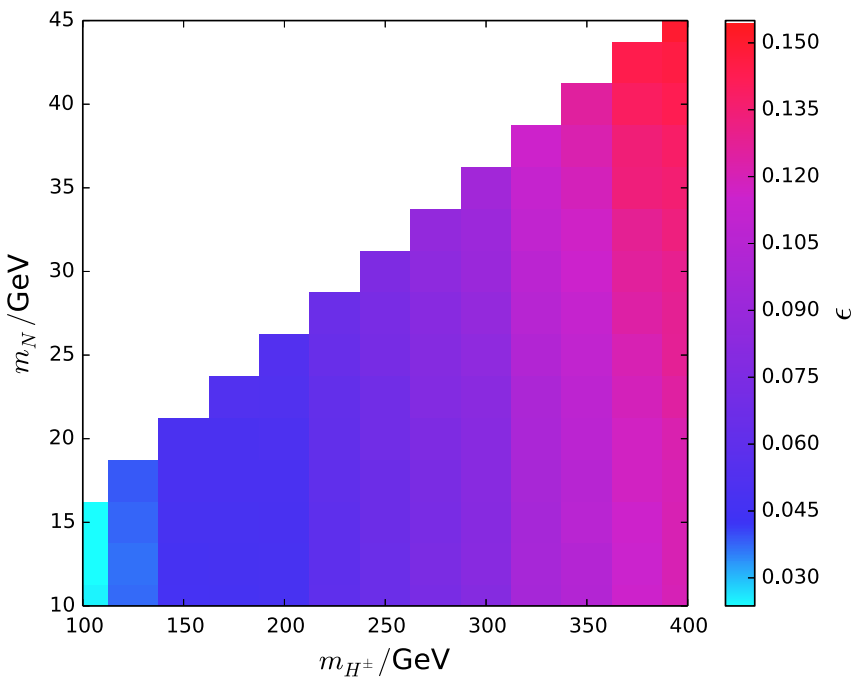

FIG. 3. Minimum $\epsilon$ for $\sqrt{2((S+B) \ln (1+S / B)-S)}=5$. The integrated luminosity is $3 \mathrm{ab}^{-1}$ at a 13 TeV proton-proton collider. $p p \rightarrow t \bar{t} \rightarrow \mu^{+} \mu^{-} b \bar{b} \nu \bar{\nu}$ contributions are included.

is listed in Table III. In Table III, we fix $m_{N}$ at $10 \mathrm{GeV}$ for the limited size of the table.

In Figs. 2 and 3, we show the minimum $\epsilon$ for the significance defined by $\sqrt{2((S+B) \ln (1+S / B)-S)}=$ 5 with a luminosity of $3 \mathrm{ab}^{-1}$ as proposed by the HL-LHC. Both the "- $1 \mathrm{~N}$-jet" and "- $2 \mathrm{~N}$-jet" results are plotted. For comparison, we also show in Fig. 4 the results for $100 \mathrm{fb}^{-1}$ of integrated luminosity, which can be reached in the near future. Here, only the "- $2 \mathrm{~N}$-jet" results are plotted. Again,

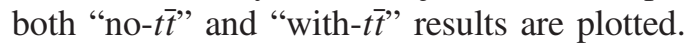

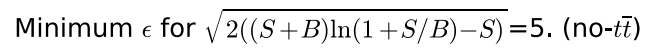

Integrated luminosity $=100 \mathrm{fb}^{-1}$, two $\mathrm{N}$-jet tagged.

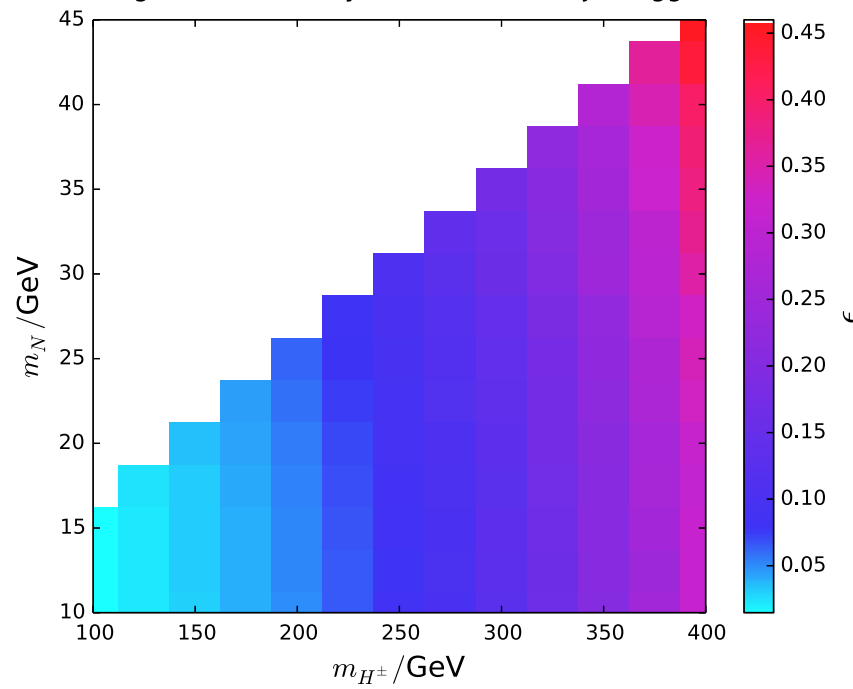

\section{DISCUSSIONS}

Same-sign charged lepton signals could appear in the case of the Majorana sterile neutrino. We should note that the isolated leptons decayed directly from the $H^{ \pm}$are definitely oppositely charged. Therefore, we should consider the charges of the leptons appearing inside the jets. We have not found any information in the literature addressing the charge identification performance in such a case. Furthermore, in the pseudo-Dirac sterile neutrino case, there should not be any significant same-sign lepton

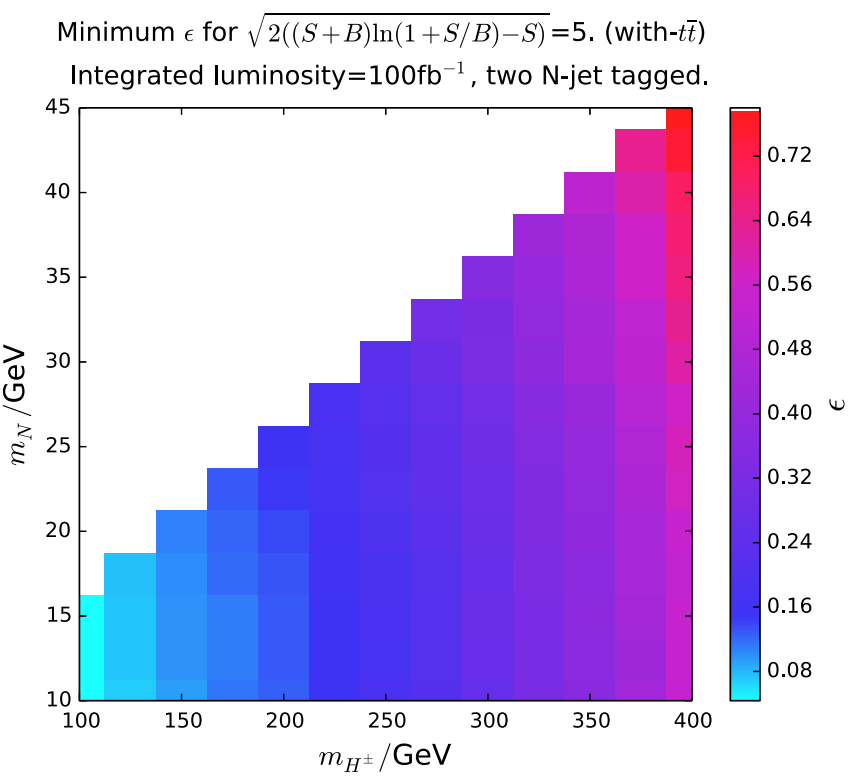

FIG. 4. Minimum $\epsilon$ for $\sqrt{2((S+B) \ln (1+S / B)-S)}=5$. The integrated luminosity is $100 \mathrm{fb}^{-1}$ at a 13 TeV proton-proton collider. In the left panel contributions from the $p p \rightarrow t \bar{t} \rightarrow \mu^{+} \mu^{-} b \bar{b} \nu \bar{\nu}$ are not included, while in the right panel they are included. 


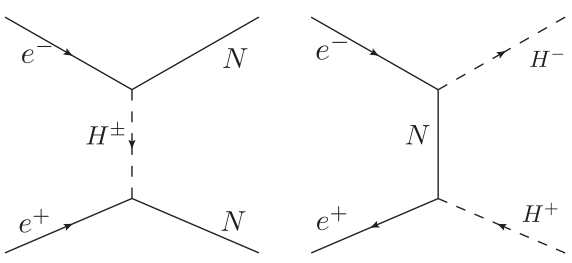

FIG. 5. $t$-channel diagrams at an $e^{+} e^{-}$collider.

signals. In order to have a safe and careful discussion, and for the convenience of transferring our results to the pseudo-Dirac cases, we did not discuss this kind of signal.

As we have mentioned, we have only calculated the results of the muon channels, but our results can be transferred to the $H^{ \pm} \rightarrow e^{ \pm} N$ channels. However, it is difficult to discriminate the electron within the $N \rightarrow e q \bar{q}$ jets. If we again let $N \rightarrow \mu q \bar{q}$ with $H^{ \pm}$still decaying to the isolated electrons, the channel $H^{ \pm} \rightarrow \mu^{ \pm} N$ is also inevitable. Therefore, a lepton-flavor-violation $e^{ \pm} \mu^{\mp}$ signal could arise, giving a rather significant signal in the case where $t \bar{t}$ backgrounds can be effectively eliminated.

Compared with the hadron-hadron colliders, an $e^{+} e^{-}$ collider provides a cleaner background. As we know, there are currently proposals for the Circular Electron Positron Collider (CEPC) [53,54], the International Linear Collider (ILC) $[55,56]$, the Compact Linear Collider (CLIC) $[57,58]$, and the $e^{+} e^{-}$project at the Future Circular Collider (FCC-ee) $[59,60]$. The CEPC and FCC-ee are circular colliders with no proposals for $\sqrt{s} \gtrsim 500 \mathrm{GeV}$ that we know of, while both the ILC and CLIC have plans to run at the $\sqrt{s} \gtrsim 1 \mathrm{TeV}$ scale.

At an $e^{+} e^{-}$collider, the sterile neutrino pairs can be directly produced through the $t$-channel diagram in the left panel of Fig. 5. This channel might be significant due to the potentially large $e-H^{ \pm}-N$ Yukawa couplings. Besides the electroweak processes similar to Fig. 1, charged Higgs bosons can also be produced through the exchange of a $t$-channel sterile neutrino, as in the right panel of Fig. 5.

In the signal-background analysis at an $e^{+} e^{-}$collider, at least one more technique can be applied to suppress the background. In fact, unlike the sterile neutrino, the semileptonic decay of $B$ mesons also produces a neutrino, and thus the missing energy arises. Due to the more precise measurement of the total missing energy and the no-pileup environment, the missing energy can also become a good kinematic tool to cut the background.

\section{CONCLUSIONS}

In this paper we have discussed a collider simulation in a particular parameter space in the $\nu$-THDM. In this model the light sterile neutrinos are the decay product of the heavy charged Higgs boson. In the $m_{N} \ll m_{H}^{ \pm}$cases, the muons appearing in the collimated decay products of the sterile neutrinos can help us discriminate the $N$ jets from the QCD jet backgrounds. We have shown that these backgrounds can be effectively suppressed. In some parameter space, a future proton-proton $3000 \mathrm{ab}^{-1}$ collider can be sensitive to the $\epsilon \lesssim 0.01$ cases. The reducible $p p \rightarrow t \bar{t}$ background plays an important role in the sensitivity. Collider designs and performances should be extensively studied before trying to eliminate this channel.

\section{ACKNOWLEDGMENTS}

The author would like to thank for Pyungwon Ko, Taoli Cheng, Peiwen Wu, and Jinmian Li for helpful discussions. This work is supported in part by the National Research Foundation of Korea (NRF) Research Grant No. NRF2015R1A2A1A05001869, and the Korea Research Fellowship Program through the National Research Foundation of Korea (NRF) funded by the Ministry of Science and ICT (2017H1D3A1A01014127).
[1] P. Minkowski, Phys. Lett. B 67, 421 (1977).

[2] T. Yanagida, in Proceedings of the Workshop on Unified Theories and Baryon Number in the Universe, Tsukuba, Japan, 1979, edited by O. Sawada and A. Sugamoto (KEK, Tsukuba, 1979), p. 95.

[3] P. Ramond, CALT-68-709, arXiv:hep-ph/9809459; M. GellMann, P. Ramond, and R. Slansky, in Supergravity, edited by P. Van Nieuwenhuizen and D.Z. Freedman (North Holland, Amsterdam, 1979), p. 315.

[4] S. Glashow, in Quarks and Leptons, edited by M. Lévy (Plenum, New York, 1980), p. 707.

[5] R. N. Mohapatra and G. Senjanovic, Phys. Rev. Lett. 44, 912 (1980).

[6] D. Wyler and L. Wolfenstein, Nucl. Phys. B218, 205 (1983).
[7] R. N. Mohapatra and J. W. F. Valle, Phys. Rev. D 34, 1642 (1986).

[8] E. Ma, Phys. Lett. B 191, 287 (1987).

[9] P. Abreu et al. (DELPHI Collaboration), Z. Phys. C 74, 57 (1997); 75, 580(E) (1997).

[10] G. Aad et al. (ATLAS Collaboration), J. High Energy Phys. 07 (2015) 162.

[11] V. Khachatryan et al. (CMS Collaboration), Phys. Lett. B 748, 144 (2015).

[12] F. F. Deppisch, P. S. Bhupal Dev, and A. Pilaftsis, New J. Phys. 17, 075019 (2015).

[13] S. Antusch, E. Cazzato, and O. Fischer, Int. J. Mod. Phys. A 32, 1750078 (2017).

[14] C. O. Dib and C. S. Kim, Phys. Rev. D 92, 093009 (2015). 
[15] Y. Cai, T. Han, T. Li, and R. Ruiz, Front. Phys. 6, 40 (2018).

[16] E. Izaguirre and B. Shuve, Phys. Rev. D 91, 093010 (2015).

[17] S. Dube, D. Gadkari, and A. M. Thalapillil, Phys. Rev. D 96, 055031 (2017).

[18] G. Anamiati, M. Hirsch, and E. Nardi, J. High Energy Phys. 10 (2016) 010.

[19] C. O. Dib, C. S. Kim, and K. Wang, Chin. Phys. C 41, 103103 (2017).

[20] A. Das, arXiv:1701.04946.

[21] A. Das, P. Konar, and A. Thalapillil, J. High Energy Phys. 02 (2018) 083.

[22] A. Das, P. S. B. Dev, and C. S. Kim, Phys. Rev. D 95, 115013 (2017).

[23] J. Kersten and A. Yu. Smirnov, Phys. Rev. D 76, 073005 (2007).

[24] A. Ibarra, E. Molinaro, and S. T. Petcov, J. High Energy Phys. 09 (2010) 108.

[25] A. Das and N. Okada, Phys. Rev. D 88, 113001 (2013).

[26] P. S. B. Dev and A. Pilaftsis, Phys. Rev. D 86, 113001 (2012).

[27] A. Das, P. S. Bhupal Dev, and N. Okada, Phys. Lett. B 735, 364 (2014).

[28] Z. Kang, P. Ko, and J. Li, Phys. Rev. D 93, 075037 (2016).

[29] A. Das, N. Okada, and D. Raut, arXiv:1711.09896.

[30] P. S. B. Dev, R. N. Mohapatra, and Y. Zhang, J. High Energy Phys. 03 (2018) 122.

[31] A. Das, N. Okada, and D. Raut, Phys. Rev. D 97, 115023 (2018).

[32] P. Cox, C. Han, and T. T. Yanagida, J. High Energy Phys. 01 (2018) 037.

[33] E. Accomando, C. Coriano, L. Delle Rose, J. Fiaschi, C. Marzo, and S. Moretti, J. High Energy Phys. 07 (2016) 086.

[34] P. S. Bhupal Dev and R. N. Mohapatra, Phys. Rev. Lett. 115, 181803 (2015).

[35] A. Das, N. Nagata, and N. Okada, J. High Energy Phys. 03 (2016) 049.

[36] E. Ma, Phys. Rev. Lett. 86, 2502 (2001).

[37] S. Gabriel and S. Nandi, Phys. Lett. B 655, 141 (2007).

[38] S. M. Davidson and H. E. Logan, Phys. Rev. D 80, 095008 (2009).

[39] E. Bertuzzo, Y.F. Perez G., O. Sumensari, and R.Z. Funchal, J. High Energy Phys. 01 (2016) 018.

[40] N. Haba and K. Tsumura, J. High Energy Phys. 06 (2011) 068.
[41] C. Guo, S.-Y. Guo, Z.-L. Han, B. Li, and Y. Liao, J. High Energy Phys. 04 (2017) 065.

[42] M. Mitra, R. Ruiz, D. J. Scott, and M. Spannowsky, Phys. Rev. D 94, 095016 (2016).

[43] O. Mattelaer, M. Mitra, and R. Ruiz, arXiv:1610.08985.

[44] G. C. Branco, P. M. Ferreira, L. Lavoura, M. N. Rebelo, M. Sher, and J. P. Silva, Phys. Rep. 516, 1 (2012).

[45] Y.-L. Tang and S.-h. Zhu, Phys. Rev. D 96, 055022 (2017).

[46] A. Alloul, N. D. Christensen, C. Degrande, C. Duhr, and B. Fuks, Comput. Phys. Commun. 185, 2250 (2014).

[47] J. Alwall, R. Frederix, S. Frixione, V. Hirschi, F. Maltoni, O. Mattelaer, H. S. Shao, T. Stelzer, P. Torrielli, and M. Zaro, J. High Energy Phys. 07 (2014) 079.

[48] T. Sjöstrand, S. Ask, J. R. Christiansen, R. Corke, N. Desai, P. Ilten, S. Mrenna, S. Prestel, C. O. Rasmussen, and P. Z. Skands, Comput. Phys. Commun. 191, 159 (2015).

[49] J. de Favereau, C. Delaere, P. Demin, A. Giammanco, V. Lemaître, A. Mertens, and M. Selvaggi (Delphes 3 Collaboration), J. High Energy Phys. 02 (2014) 057.

[50] CMS Collaboration, Technical Report No. CMS-PAS-PFT10-003, http://cds.cern.ch/record/1279347.

[51] CMS Collaboration, Performance of muon identification in $p p$ collisions at $\sqrt{s}=7 \mathrm{TeV}$, Report No. CMS-PAS-MUO10-002.

[52] E. James, Y. Maravin, M. Mulders, and N. Neumeister, Muon identification in CMS, Report No. CERN-CMSNOTE-2006-010.

[53] CEPC-SPPC Study Group, CEPC-SPPC Preliminary Conceptual Design Report. 1. Physics and Detector, IHEP-CEPCDR-2015-01, IHEP-TH-2015-01, IHEP-EP-2015-01.

[54] CEPC-SPPC Study Group, CEPC-SPPC Preliminary Conceptual Design Report. 2. Accelerator, IHEP-CEPCDR-2015-01, IHEP-AC-2015-01.

[55] H. Baer et al., arXiv:1306.6352.

[56] C. Adolphsen et al., arXiv:1306.6328.

[57] L. Linssen, A. Miyamoto, M. Stanitzki, and H. Weerts, arXiv:1202.5940.

[58] M. Aicheler, P. Burrows, M. Draper, T. Garvey, P. Lebrun, K. Peach, N. Phinney, H. Schmickler, D. Schulte, and N. Toge, Report No. CERN-2012-007.

[59] D. d'Enterria, in Proceedings of the 17th Lomonosov Conference on Elementary Particle Physics, Moscow, Russia, 2015, edited by A. I. Studenikin (World Scientific, Singapore, 2017), p. 182.

[60] D. d'Enterria, Frascati Phys. Ser. 61, 17 (2016). 